\title{
Maximizing the Reliability of a Full-Automatic ECG-Waveforms Delineating Algorithm Using Extensive ECG Databank
}

\author{
Antoun Khawaja ${ }^{1}$ \\ ${ }^{1}$ Khawaja Medical Technology GmbH, Gräfelfing near Munich, Germany
}

\begin{abstract}
Due to the significance of ECG delineation and measurements including $Q T$ measurement for drug safety, the objective of this work is to compare the results of manual annotated $Q T$ and $R R$ interval measurements of a huge databank with the results of the fully-automated algorithm presented in [3] for validation purposes not only in clinical studies and cardiac safety, but also in all kind of cardiac applications. The differences between the results of the algorithm and the golden manual-annotated reference values are very low. This gives a strong indicator for the reliability of the full-automated delineation program.
\end{abstract}

\section{Introduction}

There is in fact a large interest in accurate ECG delineation and measurements not only because they play a role in further ECG analysis, but also because they are considered themselves important biomarkers for making decisions. Some ECG measurements, like RR intervals, QT/QTc interval prolongation, are even considered as the standard surrogate biomarker for cardiac drug safety as stated by ICH clinical evaluation guideline E14 [1] . Manual, semi-automatic and automatic techniques are used to find the fiducial points of ECG signals and to measure their corresponding intervals including QT and RR. In order to ensure reliable and precise detection and measurement, the manual and semi-automatic methods have been employed so far. Accordingly, many efforts have been done to validate the automatic methods against the manual and semiautomatic ones [4],[5], [6] and [7] and furthermore to compare the output performance of different algorithms for $\mathrm{T}$ wave delineation [8] . The objective of this work is to validate the precision of the delineation program published in [3] using huge number of manually annotated ECG signals. This databank contains more than seventy thousand 12- lead resting ECG records from Thorough QT (TQT) trials and several late-phase clinical studies. For each ECG signal, a full corresponding set of measurement including QT interval and RR interval is annotated manually by var- ious cardiologists and certified medical experts. Those annotations are considered as golden reference for this work. The measurements obtained by the delineation algorithm [3] using the databank under study were compared with golden reference including RR intervals and QT intervals. The mean, median and standard deviation of the differences between the full-automated program and the golden reference for all relevant interval and segment measurements are very low. For QT interval measurements, the mean, median and standard deviation of the differences are less than 2, 3 and $10[\mathrm{~ms}]$, respectively.

\section{Methods}

\subsection{Database}

The database used in this study contains more than seventy thousand 12-lead 10-second resting ECG signals from Thorough QT trials and several late-phase clinical studies. The data were sampled with $500[\mathrm{~Hz}]$ and $1000[\mathrm{~Hz}]$ and they were completely anonymized. No ECG signal employed for this validation test has been modified or manipulated by de-noising or filter methods. A set of measurement including QT interval and RR interval is annotated manually by certified cardiologists or by medical experts for each ECG record. The selection of the respective observed lead and heart beats has been done according to the corresponding study protocol during the clinical study.

\subsection{Delineation methodology}

The delineation process consists of four main steps (figure 1) as follows:

\subsubsection{Preprocessing}

ECG signals under study are converted all to 500 [Hz]. No digital filter is used in this step for muscle and moving artifact, power-line interference, distortion from misconnected electrodes, or baseline wandering. 


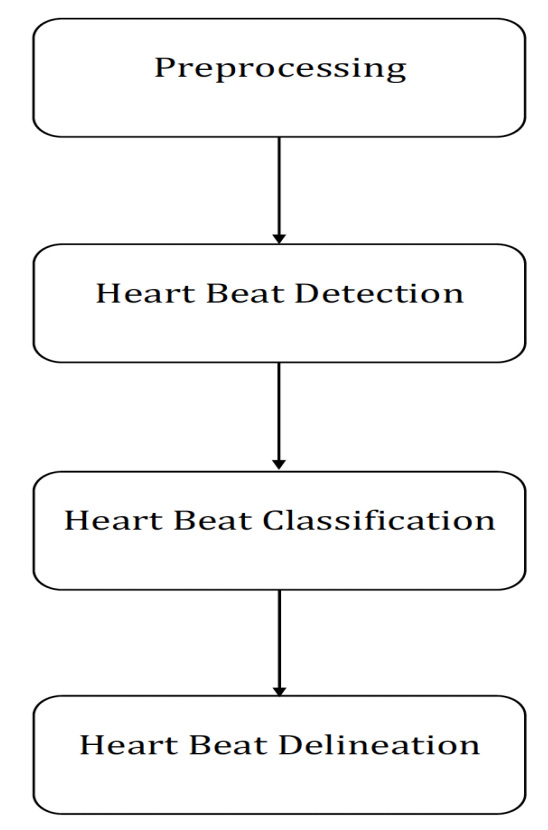

Figure 1. The process delineation methodology along with the main four steps.

\subsubsection{Heart beat detection}

In this step heart beats of the input ECG signal in every channel are detected. The methodology of identifying ECG heart beats is developed based on time-frequency analysis [2]. Besides, it carries out numbers of plausibility checks to ensure the accurate detection of the physiological heart cycles. This algorithm is validated using MIT-BIH Arrhythmia Database, AHA Series1 and AHA Series 2 and obtained sensitivity and specificity values greater than $99.7 \%$ in both measures and in all databases. This algorithm provides, in addition to heart beat locations, coarse position of QRS onset and QRS offset for each cycle. Figure 2 shows an example for heart beat detection done on a randomly-chosen real-world ECG signal using this algorithm.

\subsubsection{Heart beat classification}

Detected heart beats are classifies in this step. The methodology of classifying ECG heart beats is developed based on machine-learning techniques [2]. The algorithm can classify detected heart beats into five main classes, namely Normal for normal beats, $P V C$ for premature ventricular contraction beats, $P S C$ for premature supraventricular contraction beats, Paced for paced beats and Unclassified for beats that cannot be classified. This algorithm is validated using MIT-BIH Arrhythmia Database,

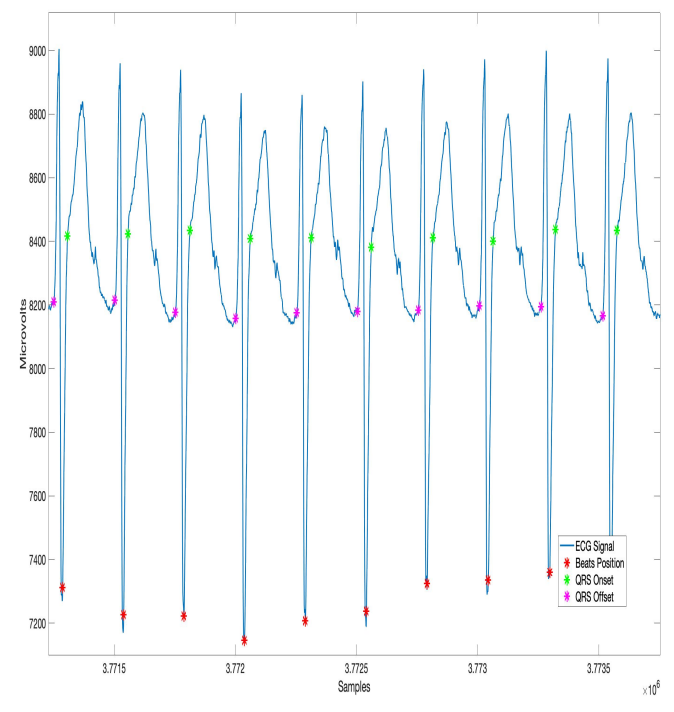

Figure 2. An example for heart beat detection done on a randomly-chosen real-world ECG signal.

AHA Series1 and AHA Series 2 and obtained sensitivity and specificity values greater than $93.5 \%$ and $96.5 \%$ in all databases, respectively. Figure 3 shows an example of a 12-channel ECG signal where the all heart beats are localized and classified.

\subsubsection{Heart beat delineation}

In the step, the algorithm responsible for detecting the essential fiducial points and measuring the segments and intervals of each detected heart beat is employed [2] The fiducial points localized here are $\mathrm{P}$ wave onset, $\mathrm{P}$ wave offset, QRS complex onset, QRS complex offset and T wave offset. Whereas, the segments and intervals are PQ / PR segment, ST segment, PR interval, QRS interval/duration and QT interval. This algorithm is validated and tested according to the procedure and databases described in the standard IEC 60601-2-25:2011. All relevant requirements of the standard have been fulfilled by the delineation algorithm. The delineation results illustrates a high degree of agreement with the reference annotations done in CTS and CSE and the delineation results provided by the Heart Beat Delineation Algorithm [3]. Figure 4 shows the results of the main fiducial points detected by the algorithm on a randomly chosen signal and beat. Figure 5 shows the beatto-beat results of the main fiducial points detected by the algorithm on a randomly chosen single-lead signal. The ECG signal is illustrated on the upper side of the figure. Whereas the lower side represents the main fiducial points for every heart beat, namely '1', '2', '3', '4', '5' and '6' 


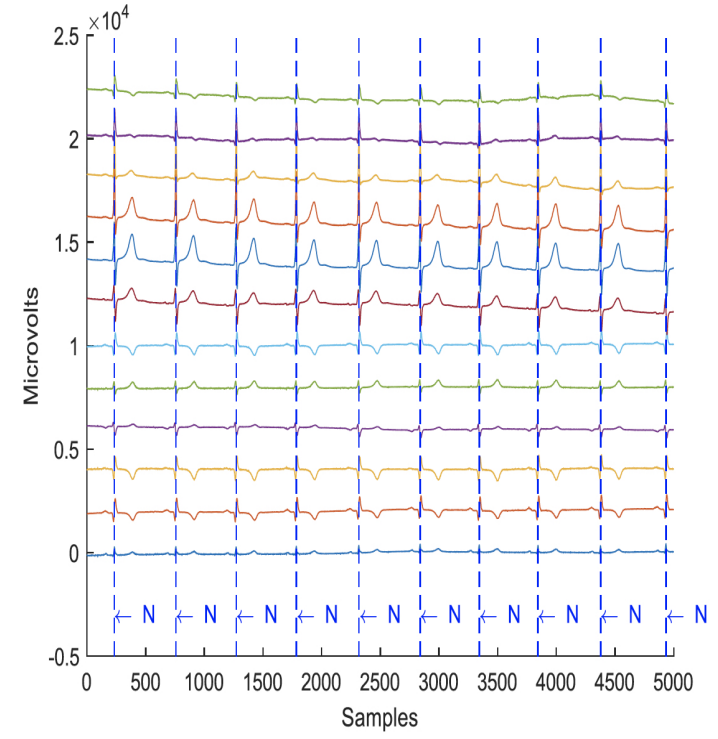

Figure 3. An example of a 12-channel ECG signal where the all heart beats are localized and classified.

for P wave onset, $\mathrm{P}$ wave offset, QRS complex onset, $\mathrm{R}$ peak, QRS complex offset and T wave offset, respectively.

\subsection{Validation methodology}

The measurements obtained from the fully-automated algorithm, namely QT and RR intervals were compared with reference measurements annotated by the experts and certified cardiologists on the used database. The results of the validation process are represented in this work by the differences and absolute differences between the results of these two delineation techniques as well as the mean Mean, standard deviation STD and median Median of these differences for all records under study.

\section{Results}

\subsection{RR interval measurement}

The Mean, standard deviation STD and Median values for the differences and absolute differences between the results of the fully-automated algorithm and the manual reference technique for RR interval using the used databank are illustrated in 1 . Since the mean of the RR difference between the algorithm and reference value is around two times sampling interval, namely 4 [ms], this difference can be considered as low.

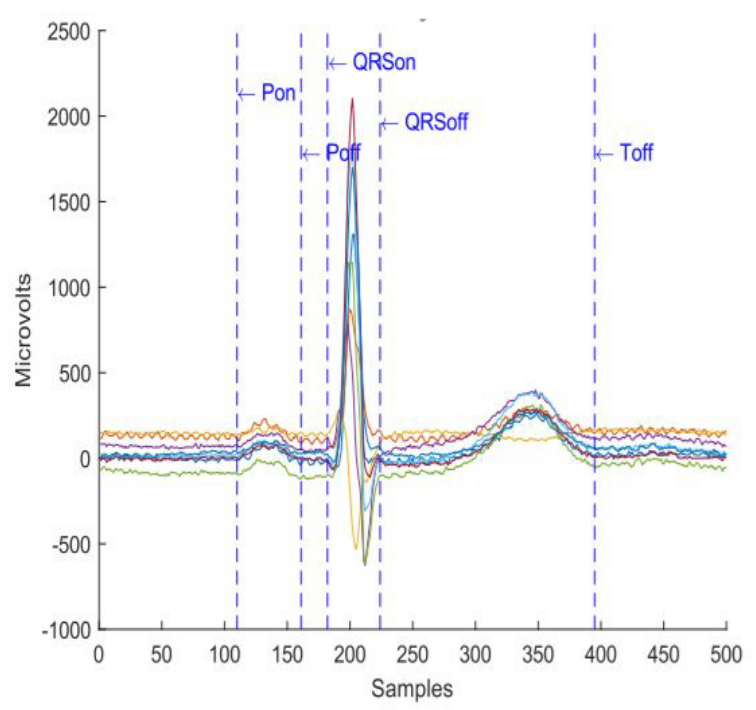

Figure 4. The results of the main fiducial points detected by the algorithm on a randomly chosen signal and beat.

Table 1. The Mean, standard deviation STD and Median values for the differences and absolute differences between the results of the fully-automated algorithm and the manual reference technique for RR interval using the used databank.

\begin{tabular}{lcr}
\hline \hline Mean & Median & Standard Deviation \\
\hline less than & less than & less than \\
$4 \mathrm{~ms}$ & $4 \mathrm{~ms}$ & $7 \mathrm{~ms}$ \\
\hline \hline
\end{tabular}

\subsection{QT interval measurement}

The Mean, standard deviation STD and Median values for the differences and absolute differences between the results of the fully-automated algorithm and the manual reference technique for QT interval using the used databank are illustrated in table 2. The mean of the QT difference between the algorithm and reference value is very low, since it is smaller than the mean signal digitization error, namely $1[\mathrm{~ms}]$ and since the median QT difference is identical to the sampling interval, namely $2[\mathrm{~ms}]$.

\section{Discussion and conclusions}

The delineation precision for single-lead and single-beat measurements of the QT and RR interval in the algorithm described in [3] has been investigated and validated in this work. It is done using a very large database containing more than seventy thousand manually-annotated 12-lead ECG records from cardiac safety studies. The measure- 


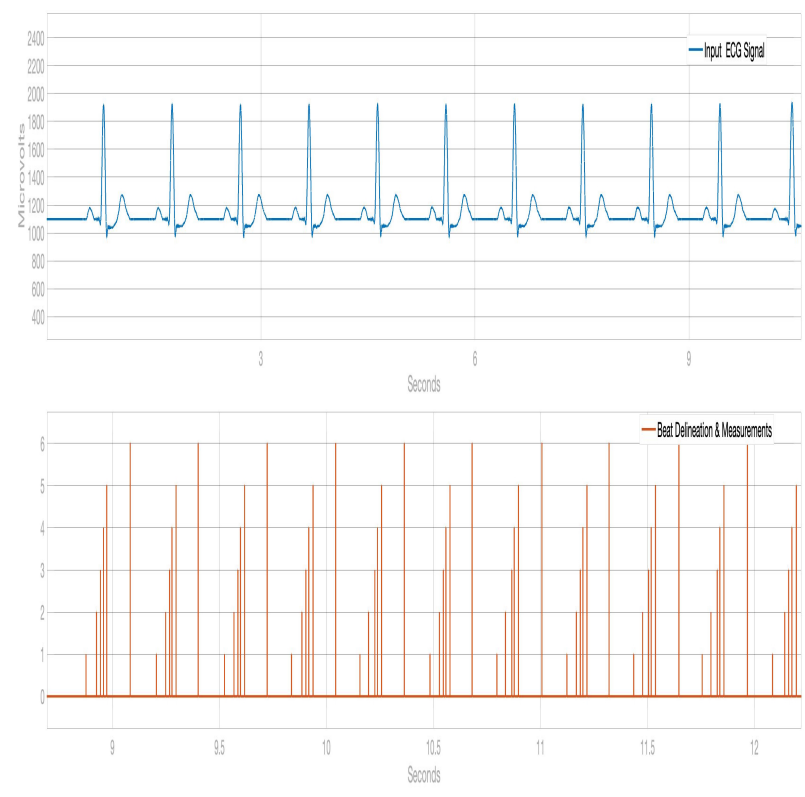

Figure 5. The beat-to-beat results of the main fiducial points detected by the algorithm on a randomly chosen single-lead signal. The ECG signal is illustrated on the upper side. Whereas the lower side represents the main fiducial points for every heart beat, namely '1', '2', '3', '4', '5' and '6' for P wave onset, $\mathrm{P}$ wave offset, QRS complex onset, R peak, QRS complex offset and T wave offset, respectively.

Table 2. The Mean, standard deviation STD and Median values for the differences and absolute differences between the results of the fully-automated algorithm and the manual reference technique for QT interval using the used databank.

\begin{tabular}{lcr}
\hline \hline Mean & Median & Standard Deviation \\
\hline less than & less than & less than \\
$2 \mathrm{~ms}$ & $3 \mathrm{~ms}$ & $10 \mathrm{~ms}$ \\
\hline \hline
\end{tabular}

ment obtained by the experts in these studies has been considered as golden reference for this work. The low mean differences between the reference and automatic measure- ments of QT and RR intervals expresses that the algorithm described in [3] is a useful tool in all kind of ECG applications including resting ECG, Holter analysis, stress ECG, ECG monitoring and for the evaluation of cardiac safety in clinical trials. These findings can be interpreted as an indicator of the reliability of our algorithm in the fullautomatic measurements of ECG waveforms.

\section{References}

[1] B Darpo, T Nebout, PT Sager, "Clinical evaluation of qt/qtc prolongation and proarrhythmic potential for nonantiarrhythmic drugs: the international conference on harmonization of technical requirements for registration of pharmaceuticals for human use", e14 guideline, J Clin Pharmacol 2006;46:498-507.

[2] A Khawaja, "A Novel Algorithm for Full-Automatic ECG Interpretation and Diagnostics", in Proc. Computers in Cardiology, vol. 45, ISSN: 2325-887X DOI: 10.22489/CinC.2018.229, 2018.

[3] A Khawaja, "A Novel Algorithm for Full-Automatic Multipurpose ECG Delineation," in Proc. Computers in Cardiology, vol. 45,ISSN: 2325-887X DOI: 10.22489/CinC.2018.209, 2018.

[4] JS Sahambi, SN Tandon, RKP Bhatt, "An automated approach to beat-by-beat qt-interval analysis", 2000;19:97-101.

[5] N Hughes, "Probabilistic Models for Automated ECG Interval Analysis", University of Oxford, 2006.

[6] NB McLaughlin, RWF Campbell, A Murray, "Accuracy of automatic qt measurement techniques.", in Proc. Computers in Cardiology, 1993;863-866.

[7] SH Meij, P Klootwijk, J Arends, JRTC Roelandt, ”An algorithm for automatic beat-to-beat measurement of the qt interval.", in Proc. Computers in Cardiology, 1994;597-600.

[8] T Baas, F Gravenhorst, R Fischer, A Khawaja, O Dossel, "Comparison of three t-wave delineation algorithms based onwavelet filterbank, correlation and pca", Computing in Cardiology, 2010;361-364.

Address for correspondence:

Dr. Antoun Khawaja

Am Haag 8, 82166 Gräfelfing near Munich, Germany

Antoun.Khawaja@khawaja-medtech.com 